\title{
On Correcting the Intrusion of Tracing Non-deterministic Programs by Software
}

\author{
Florin Teodorescu ${ }^{1}$ and Jacques Chassin de Kergommeaux ${ }^{2}$ \\ 1 Computer Science Department, "Politehnica" University of Bucharest, 313 Splaiul \\ Independenţei, Bucharest, Romania \\ 2 LMC-IMAG, B.P. 53, F-38041 Grenoble Cedex 9, France
}

\begin{abstract}
This paper describes a performance evaluation technique of parallel programs based on software tracing. The interest of the proposed method is to enable post-mortem correction of the intrusion of software tracing of non deterministic programs (probe effect), by use of RECORD -REPLAY debugging techniques. In the first phase (RECORD), a primary trace is collected with a very low perturbation. This primary trace is used to force deterministic re-executions of parallel programs during subsequent replayed phases. The method proposed in the paper takes the initial RECORD execution as a reference and collects performance traces during a replayed execution. The dates of performance traces are then corrected post mortem in order to compensate the intrusion caused by the performance tracing and by the deterministic re-execution mechanism.
\end{abstract}

\section{Introduction}

Tracing is a monitoring technique based on the recording of observable events of interest in a trace, followed by an analysis of the trace to extract performance indices or visualize the execution of a program [5,4]. Among the possible tracing techniques: hardware, hybrid and software [4], software tracing is deemed more general and portable. One of the most important drawbacks of software tracing is the perturbation induced by tracing. This perturbation is caused by several types of intrusions [10]:

Direct intrusion: the recording of each event takes some amount of time, and thus delays the execution of all following events.

Indirect intrusion: software tracing induces other types of intrusion, more difficult to detect and to estimate. For example, the insertion of tracing instructions in a program can hinder some compiler optimizations or alter the behavior of caches.

Indirect intrusion is very difficult to estimate while several methods correct direct intrusion post mortem very precisely $[8,10]$. However these methods cannot be applied to parallel programs behaving non deterministically (see figure 1) where only a conservative approximation can be done. 


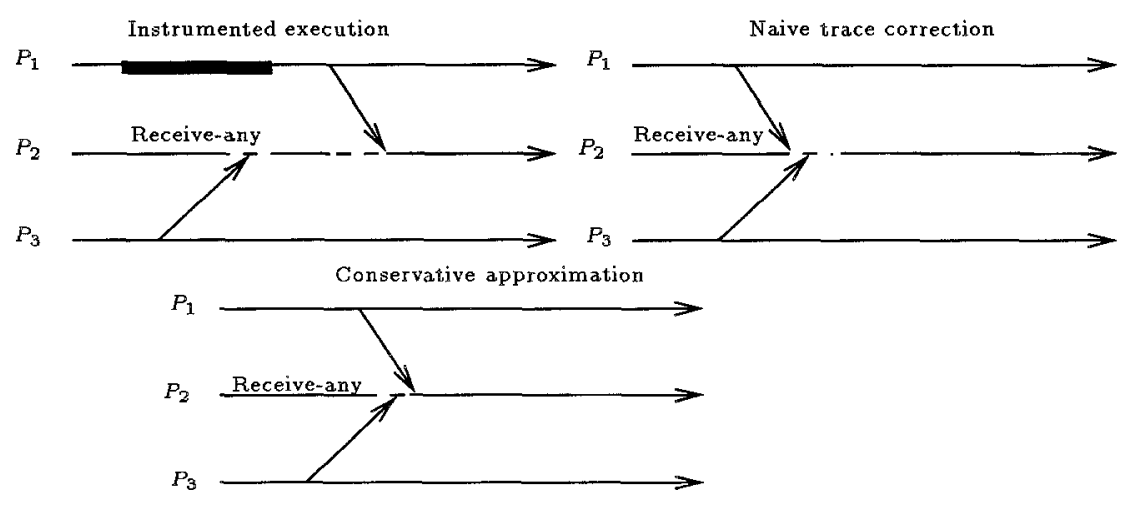

Fig. 1. Intrusion correction of non deterministic programs

In this example, the execution of $P_{1}$ was deeply perturbated by software tracing. If the emission date of the message from $P_{1}$ to $P_{2}$ were corrected naively, the transmission duration remaining constant, the order of reception of messages from $P_{1}$ and $P_{3}$ by $P_{2}$ would be inverted on the corrected trace. However, it would not be possible to reconstruct post mortem what would have occurred in that case on $P_{2}$ and a conservative approximation had to be used in [8] instead.

This article proposes a method to correct the direct intrusion of software tracing of non deterministic programs. The aim of the method is to reconstruct post mortem the dates that the recorded events would have in a very slightly perturbated execution. Tracing is performed in two phases, using a low intrusive debugging tool [2] based on the instant replay technique [6].

Primary traces are collected during a first phase, low intrusive RECORD execution. These traces are used in a second phase (REPLAY execution), to force the program execution to be identical to the one of the previous phase. Performance traces are collected during the deterministic REPLAY execution. Traces collected during both previous phases are then passed to a post mortem correction tool. Both intrusions induced by the trace instrumentation and the re-execution mechanism are then corrected, in order to retrieve the time estimates of the RECORD execution events. The incentive of this work was the result of systematic measurements of the RECORD execution overhead which was found hardly noticeable [3].

Although our work is guided by the same principle as the techniques presented by É. Maillet in [8] and by A. D. Malony in [10], our main improvement consists in considering the event ordering alteration and thus, the design of the evaluation mechanism over an existent deterministic re-execution mechanism. Our approach is inspired by the method used by É. Leu and A. Schiper [7] to visualize parallel programs during REPLAY executions: they use the time stamps recorded during a RECORD execution to generate the time stamps of the visual events. Compared to our method, the method of É. Leu and A. Schiper is in- 
teractive during the REPLAY phase but requires to save more information in the RECORD phase. However the objectives of both tools are different since they are interested in interactive visualization and debugging while we aim at evaluating performances: our post mortem correction method makes it possible to implement a very precise global time [9] which avoids most causal incoherencies that could appear otherwise. The monitoring tool described in [11] shares some of our objectives while being interactive. However the heavy perturbations caused to parallel executions by the intrusion correction algorithm seem to compromise the objective of being non intrusive.

The organization of this article is the following. After a presentation of the ATHAPASCAN programming environment in the framework of which the proposed technique was developed, the software tracer and the intrusion correction algorithms are briefly described.

\section{The Athapascan programming environment}

\subsection{Athapascan programming model}

The techniques described in this article were developed for the early version (ATHAPASCAN-0a) of the ATHAPASCAN programming model [1] where the execution of parallel programs is performed by a set of identical virtual processors operating asynchronously. Parallelism is expressed by blocking and non-blocking remote procedure calls (requests), thereby hiding the underlying communication protocols under the parameters and results transmission mechanisms. Each virtual processor includes several Entry Points, which are the targets of remote procedure calls (fig. 2). In this model, no other communication than parameters and results transmissions is available. A remote procedure call results in the execution of a light-weight process (thread) within the virtual processor holding the target entry point. This thread may in turn create new threads by issuing remote procedure calls. Several threads execute concurrently within each virtual processor to hide the latency of communications in parallel systems.

\subsection{Execution replay in Athapascan}

The principle of the control driven replay is to record the order of accesses to shared resources. Classical implementations of Instant Replay record the order of accesses to shared variables [6] or system-level primitives for passing messages [7]. In the ATHAPAsCAN model, shared resources are entry points, accessed by requests. Each call to an entry point results in a typical sequence of such "abstract" events. Figure 2 represents a complete sequence of events generated by a call to an entry point, from the request emission (event a) to the result receipt (event d) passing through the request receipt (event b) and the result emission (event c). A replay can be driven by forcing the execution order of request receipts (event b). Since an entry point controls racing requests, it can be responsible for recording the order in which it serves incoming requests (event $\mathbf{b}$ ). It was proved [2] that these records are sufficient to be able to replay ATHAPASCAN programs deterministically. 


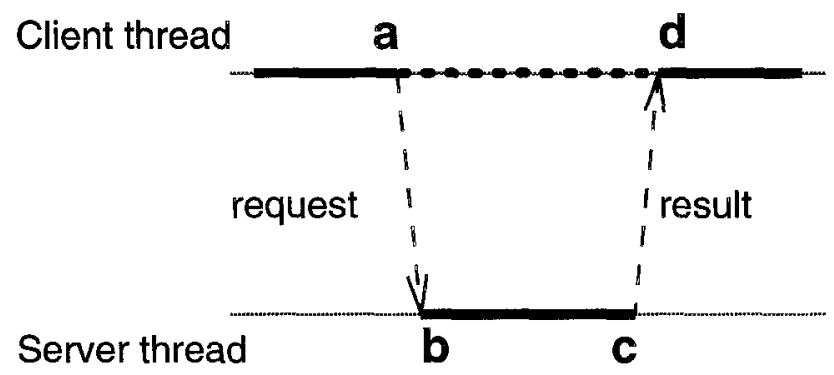

Fig. 2. Sequence of events during a call to an entry point.

\section{Software tracing in Athapascan}

Performance evaluation of ATHAPASCAN programs includes several phases:

- initial RECORD execution of the program to be evaluated. During this execution, each entry point records the order of service of incoming requests (event $\mathbf{b}$ above). In addition to a unique request identifier, the physical clock value of the virtual processor spawning a new thread is recorded.

- traced REPLAY execution, where performance data is recorded for each traced event. In addition this REPLAY execution records enough sample data to estimate post mortem a global time in the parallel system, from the local physical clocks of each processor [9]. At the end of the parallel execution, all the local dates are replaced by the corresponding global time values to avoid causal incoherencies that could be caused otherwise by the differences between the local clocks of the processors.

- post mortem correction of traces by a discrete event simulator. The main goal of this phase is to replace the dates of the events of the REPLAY traces by estimates of the dates of the corresponding events during the RECORD execution, to eliminate the intrusive effects of the REPLAY mechanism and of performance tracing.

To be able to reconstruct post mortem the execution of an ATHAPASCAN program, it is necessary to record at least the following events during the REPLAY execution: thread beginning and termination, blocking and non blocking requests and request response arrival (events $b, c, a, d$ of figure 2).

\section{Post mortem correction of performance traces}

The post mortem correction of the trace recording intrusion is performed by a trace-driven discrete event simulator which reconstructs the execution of a parallel application at the abstraction level of the events recorded during the REPLAY execution and corrects direct and REPLAY intrusions. 


\subsection{Invariance hypothesis}

The trace correction is based on the following invariance hypothesis:

The durations of the computation and communication phases are invariant between the RECORD and REPLAY executions.

\subsection{Thread beginning events}

At the beginning of a thread execution, the deterministic reexecution mechanism controls the event ordering by inserting, if necessary, time delays before spawning a new thread. This insertion of time delays apparently increases the communication time of the corresponding requests. The order of the events is preserved, no matter if the total REPLAY execution time increases. However, we cannot make any assumption on the dependence between the time stamps of blocking/non-blocking request and thread beginning events, as the communication time invariance hypothesis (4.1) does not hold in this particular case.

This explains why the trace recording mechanism of the RECORD phase had to be slightly modified to store, in the same primary traces, the physical date of each thread beginning event. These thread beginning events define correlation points. In such a point, since both RECORD and REPLAY dates are available, the real RECORD date can be substituted to the estimated one (if it does not violate causal or multithreading constraints, see section 4.4), thus resetting all previously accumulated errors to zero.

The estimated time stamp $T_{e s t}^{i, 0}$ of the beginning of the $P_{i}$ thread ( $E_{i}^{0}$ event) is in fact a copy of the RECORD time stamp, stored in the $T_{r e c}^{i, 0}$ data of the trace record (see figure 3 ).

$$
T_{e s t}^{i, 0} \leftarrow T_{r e c}^{i, 0}
$$

The perturbation induced by the tracing mechanism of the REPLAY phase does not interfere with the estimate. On the request sender's side, the perturbation induced in the $E_{j}^{k}$ event might be considered when estimating the next event of $P_{j}$. On the newly created thread's side, the intrusion of tracing the $E_{i}^{0}$ event will also be considered when its next event will be processed.

\subsection{Compensating direct intrusion}

Consider first the most simple case: the execution of a thread having processed a request without calling any other request. Hence, the only two events recorded for this thread were $E_{i}^{0}$ and $E_{i}^{1}$, the thread beginning and ending events. Using the computation time invariance hypothesis (4.1), we obtain:

$$
T_{e s t}^{i, 1} \leftarrow T_{e s t}^{i, 0}+\left(T_{r e p}^{i, 1}-T_{r e p}^{i, 0}-\operatorname{Int} t_{i}^{0}\right)
$$




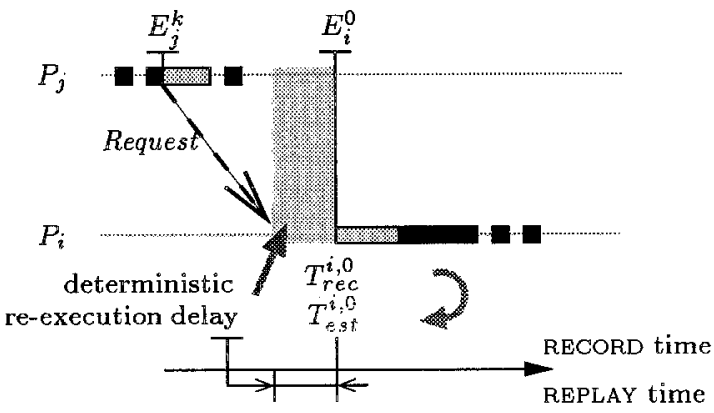

Fig. 3. Correction of a thread beginning event.

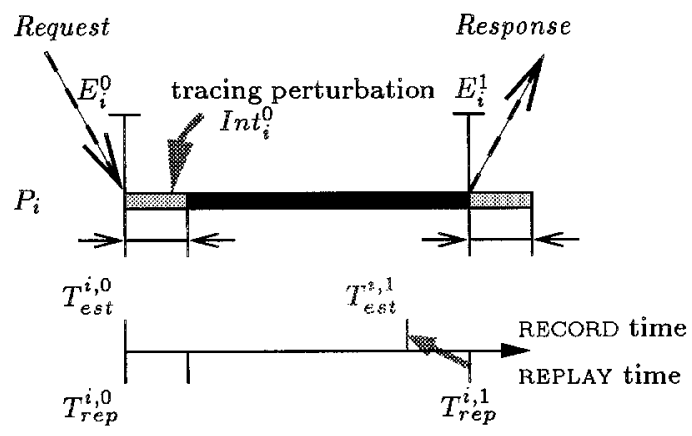

Fig. 4. Use of the invariant computation time hypothesis.

where $T_{\text {rep }}^{i, 0}, T_{\text {rep }}^{i, 1}$ are the clock values recorded during the REPLAY execution, Int $t_{i}^{0}$ is the intrusion induced by tracing the previous event $E_{i}^{0}$, recorded in the $E_{i}^{1}$ event record, while $T_{e s t}^{i, 0}$ and $T_{e s t}^{i, 1}$ are the corresponding dates estimated for the reference RECORD execution (see figure 4).

Consider now that during its execution the thread $P_{j}$ sent a request which was processed by the thread $P_{i}$. In order to enforce a deterministic re-execution, the replay mechanism might have delayed the beginning of $P_{i}$. Hence, we cannot suppose that the delay spent by $P_{j}$, between the moment the request was sent and the moment the response was received, is invariant between the RECORD and the REPLAY executions (hypothesis 4.1 does not hold for idle time). Therefore we cannot use the previous algorithm to estimate the time-stamps of response arrival events.

In this case, we take as a basis of estimated time the last date estimated for the callee thread (fig. 5). Doing so, we use the communication time invariance hypothesis (4.1), since only a communication delay separates the thread ending event of $P_{i}$ 's and the response arrival event of $P_{j}$.

$$
T_{e s t}^{j, k} \leftarrow T_{e s t}^{i, k^{\prime}}+\left(T_{r e p}^{j, k}-T_{r e p}^{i, k^{\prime}}\right)
$$




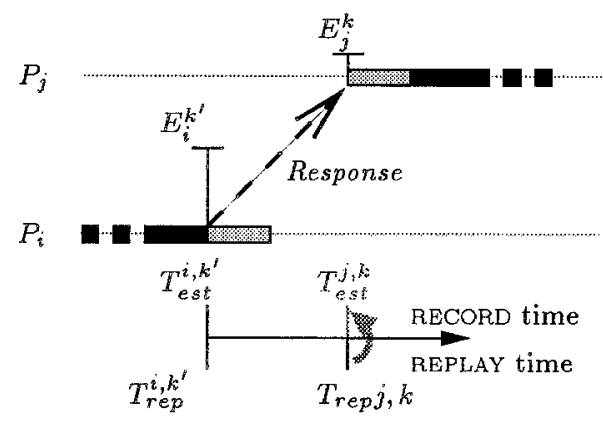

Fig. 5. Estimating the date of a response arrival event.

We should notice that no direct perturbation is considered in this equation. The first one, induced by the tracing of event $E_{i}^{k^{\prime}}$ is overlapped by the communication time. The second one, induced by the $E_{j}^{k}$ event will affect only the estimate of the time stamp of the next event of $P_{j}$.

\subsection{Incoherence handling}

Two types of incoherences may appear in the programs corrected using the (1), (2) and (3) equations only:

Causal incoherences result from the lack of control of the behavior of the operating system by the tracer. For example, a suspension of the virtual processor (system process) holding an emitting thread by the operating system, during the REPLAY execution, may lead to a violation of the invariance hypothesis (4.1). In such a case, the duration of the computation phase concerned by the suspension can be longer in the REPLAY than in the RECORD execution, leading to an estimated date of emission lower than the corresponding estimated date of reception.

Multi-threading incoherences appear when the non preemptive behavior of the thread kernel used in the ATHAPASCAN implementation is violated by some simulated threads, because of an insufficient precision of the statistical global clock mechanism [9].

Lack of space prevents us from explaining the algorithms that were designed and implemented to cope with these incoherences and to approximate as much as possible, with the corrected events dates, the dates of the corresponding RECORD events.

\section{Conclusion and future work}

This article describes a technique to correct post mortem the intrusion of software performance tracing, for programs behaving non deterministically. The technique is based on the use of an Instant Replay implementation whose recording phase 
introduces a very low overhead with respect to non instrumented executions. It is possible to correct post mortem performance traces registered during a deterministically replayed execution, in order to reconstruct the dates of the events in the corresponding, slightly perturbated RECORD execution.

This mechanism was implemented on a 32 nodes IBM SP1 parallel computer linked by an ethernet communication layer. The first experimental results are encouraging and need to be confirmed by more systematic experiments, using the methodology described in [3]. Future work includes adapting the described techniques to the evolutions of the ATHAPASCAN programming model, to be able to deal with preemptive thread kernels in particular [1].

\section{References}

1. J. Briat, I. Ginzburg, M. Pasin, and B. Plateau. Athapascan runtime: Efficiency for irregular problems. In Proceedings of EuroPar'97. Springer-Verlag, August 1997.

2. A. Fagot and J. Chassin de Kergommeaux. Formal and experimental validation of a low overhead execution replay mechanism. In Proceedings of EuroPar'95, pages 167-178. Springer-Verlag, August 1995.

3. A. Fagot and J, Chassin de Kergommeaux. Systematic assessment of the overhead of tracing parallel programs. In E.L. Zapata, editor, Proceedings of the 4th Euromicro Workshop on Parallel and Distributed processing, PDP'96, Braga, January 1996. IEEE/CS.

4. J.K. Hollingsworth, J. E. Lumpp, and B. P. Miller. Techniques for performance measurement of parallel programs. Parallel computers: theory and practice, pages $225-240,1994$.

5. E. Kraemer and J. T Stasko. The visualization of parallel systems: an overview. Journal of Parallel and Distributed Computing, 18(2):105-117, June 1993.

6. T.J. LeBlanc and J.M. Mellor-Crummey. Debugging Parallel Programs with Instant Replay. IEEE Transactions on Computers, C-36(4):471-481, April 1987.

7. E. Leu and A. Schiper. Execution replay: a mechanism for integrating a visualization tool with a symbolic debugger. In Y. Robert et al, editors, CONPAR 92 VAPP V, volume 634 of $L N C S$. Springer-Verlag, September 1992.

8. É. Maillet. Le traçage logiciel d'applications parallèles : conception et ajustement de qualité. $\mathrm{PhD}$ thesis, Institut National Polytechnique de Grenoble, France, 1996. in French.

9. E. Maillet and C. Tron. On Effciently Implementing Global Time for Performance Evaluation on Multiprocessor Systems. Journal of Parallel and Distributed Computing, 28:84-93, July 1995.

10. A. D. Malony, D. A. Reed, and H. A. G. Wijshoff. Performance measurement intrusion and perturbation analysis. IEEE Transactions on Parallel and Distributed Systems, 3(4):433-450, July 1992.

11. W. Wu, M. Spezialetti, and R. Gupta. Designing a non-intrusive monitoring tool for developing complex distributed applications. In Proc. of $2^{\text {nd }}$ IEEE Int. Conf. on Engineering for Complex Systems, Montreal, Canada, 1996. 